\title{
The heat transfer modelling for bone metastatic lesion minimization using two different cement types
}

\author{
Vânia C. C. Oliveira*, Elza M. M. Fonseca**, Jorge Belinha**, Claúdia \\ C. Rua ***, Paulo A. G. Piloto ***, Renato N. Jorge**** \\ * Centro Hospitalar e Universitário do Porto, Institute of Biomedical Sciences Abel Salazar, University of Porto (CHUP-ICBAS), Portugal, \\ vaniacoliveira@icbas.up.pt; \\ ** LAETA, INEGI, School of Engineering, Polytechnic of Porto (ISEP), Mechanical Engineering Department, Portugal, \\ elz@isep.ipp.pt,job@isep.ipp.pt; \\ ***LAETA, INEGI, Polytechnic of Institute of Bragança (IPB), Department of Applied Mechanics, Portugal, \\ claudiarua_17@hotmail.com,ppiloto@ipb.pt \\ ****LAETA, INEGI, Faculty of Engineering of the University of Porto (FEUP), Mechanical Engineering Department, Portugal, \\ rnatal@fe.up.pt
}

Special Issue: 8th Conference of the Portuguese Society of Biomechanics

\begin{abstract}
Bone tumors grow when cells divide without any control, forming a tissue mass. Bone tumors could be benign or malignant, and primary or metastatic due to systemic cancer cells dissemination. They destroy bone and lead to pathological fractures. The main objective of this work is to study the thermal effect induced by the bone cement polymerization, in the bone metastatic tumor minimization. To assess the clinical effect, it is important to test this methodology before its application and obtain sustained results. In this work, a numerical model was developed to predict the temperature distribution produced by cement polymerization. Thus, distinct tests were produced for different two cements types and amounts introduced in a cortical and spongy bone metastatic lesion, with or without an intramedullary titanium nail. The bone cement was introduced to fill in a metastatic lytic lesion area, which the main objective is playing a promising role for bone tumor necrosis due to thermal effects and biomechanical stabilization for function and pain relief. DOI: https://doi.org/10.24243/JMEB/4.4.228
\end{abstract}

$\underline{\text { Research Article }}$

2020. Published by Rational Publication.

This work is licensed under the Creative Commons Attribution 4.0 International License. To view a copy of this license, visit https://creativecommons.org/licenses/by/4.0/ or send a letter to Creative Commons, PO Box 1866,

Mountain View, CA 94042, USA

Keywords: bone tumor; metastases; thermal necrosis; cement; temperature

\section{Introduction}

Bone metastases, which are frequently diagnosed late, translate an advanced tumor stage and have a high impact in patients' quality of life and survival. After the diagnosis and tumor staging, it is important to characterize the bone tumor lesions with a specific attention regarding the identification of the size, type (osteolytic, osteoblastic, mix), location, etc. in the involved bone [1]. Tumors can destroy the spongy and cortical bone and extend to soft tissues. The

*Corresponding Vânia C. C. Oliveira Email Address: vaniacoliveira@icbas.up.pt 
bone cancer treatment is complex, and can include surgery, chemotherapy and radiotherapy, or other local or systemic treatments combinations, with the aim to cure or control the affected anatomical area.

Bone cement is widely used in orthopaedic surgeries due to their structural and physical properties, excellent biocompatibility and easy manipulation. This material has an exothermic reaction where volumetric dimension changes during the polymerization process with heat generation [2]. The amount of heat generated depends on cement mantle volume and type of cement [3]. The use of low viscosity cement poured into the bone leads to a relatively deep cement penetration, and a large volume especially when anchoring holes are used. The use of high viscosity cement is manually applied to the bone leading to a homogeneous and thinner cement mantle [3]. The high heat generated can lead to thermal necrosis of bone cells and also residual stresses formation that can affect the intramedullary systems fixation and loosening. Different authors studied the exothermic reaction of cement polymerization and reported in different publications predictive results regarding the temperature rise and residual stresses using time-dependent polymerization function [2], [4]. Others proposed empirical models for the prediction of heat generated using experimental and numerical tests [3], [5]-[9]. There are studies in the literature that point out thermal necrosis in cortical bone, usually when it reaches a temperature of $47^{\circ} \mathrm{C}$ for $1 \mathrm{~min}$ [10]-[11]. Other authors showed that temperature values above $55^{\circ} \mathrm{C}$ for a period longer than 30 seconds could cause great irreversible lesions in bone tissue [10], [12]-[13]. Eriksoon and Albrektsson [14] concluded that heating up to $47^{\circ} \mathrm{C}$ could be considered as the optimal limit that bone can withstand without necrosis. In another investigation, the thick cement showed maximum temperature approximately $45^{\circ} \mathrm{C}$ [15].

In this work, we included two different cements types (cement A and cement B) from literature. Cement type A [6] was used previously in a similar research [16] and cement type B [3] represents Palacos $R$ brand (Heraeus Medical $\mathrm{GmbH}$, Wehrheim, Germany) largely used in patients of CHUP-ICBAS. Palacos $R$ is a high-viscosity bone cement type and it is the gold standard due to its widespread use for conventional and minimally invasive surgeries. In the computational models cement was injected into an isolated mould region $(20 \mathrm{~mm}$ in depth and variable width 15 and $25 \mathrm{~mm}$ ), and the cement temperature polymerization introduced as a boundary condition. A two dimensional thermal and transient model based on finite element method was built to predict the temperature field produced by cement polymerization in a metastatic bone tumor lesion in comparison with two different bone cements types, complementing the initial research from the authors [16]. The bone cement was introduced to fill in a metastatic lytic lesion area with or without an intramedullary titanium nail. Different geometries were tested to represent metastatic lytic lesion models. For each model, the temperature field due to the cement polymerizing effect is represented for the critical time (time corresponding to the most severe thermal condition), which affects thermal necrosis and the amount of bone cells penetration. For a more efficient clinical benefit, all results were presented to stimulate a discussion regarding if the introduction of bone cement is an alternative procedure in a metastatic lytic lesion local control to add to the mechanical stabilization with an intramedullary nail. Furthermore, allows to verify the effect of using different bone cements types with thin or thick mantle amount

\section{Methods}

To analyse the effect of cementing technique, computational models were created according to average dimensions of the sub-trochanteric femoral area, using digital X-ray from patients of CHUP-ICBAS and proved from a biomechanical data control group [16]-[17]. Figure 1 a) shows a metastatic lytic lesion of the proximal left femur (subtrochanter) identified on a plain pelvic X-ray, where the patient was diagnosed with metastatic disease of a breast cancer. The right image represents other bone metastasis on the right femoral diaphysis of a male patient with clear renal cells carcinoma. Both metastatic lytic lesions have in width between 16 and $25 \mathrm{~mm}$, and $20 \mathrm{~mm}$ in depth. The size characterization of the metastatic lesions is important to introduce the same dimensions for bone cement filling minimization. Figure $1 \mathrm{~b}$ ) shows the measurement of the proximal femur (sub-trochanteric area) based on conventional pelvic X-ray of 2 patients of CHUP-ICBAS with determination of the internal and external cortical bone, spongy bone and complete femoral diaphysis diameter. On average, the measured bone geometries of 6 non-tumor patients considered for the model have an external diameter equal to $31.2 \mathrm{~mm}$ and a cortical thickness of $7.35 \mathrm{~mm}$. 


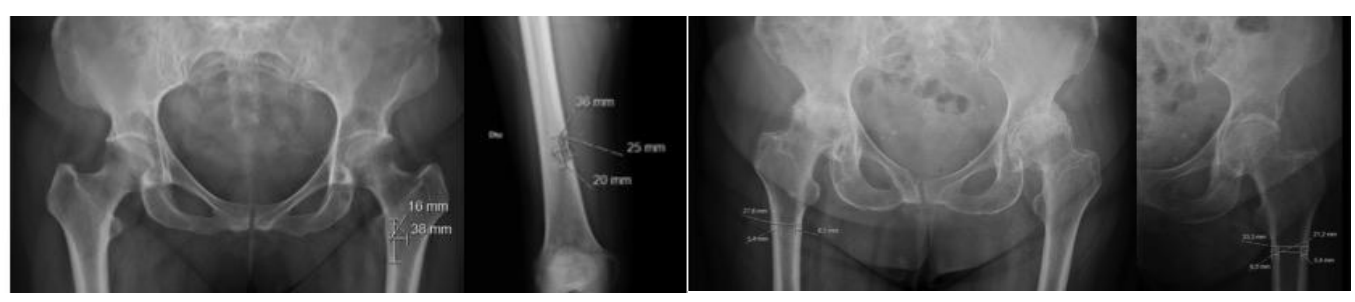

Fig.1 (a) Metastatic lytic lesion of the proximal left femur and on the diaphysis of the right femur. b) Measurements on control group patients

Figure 2 shows a conventional A-P and lateral X-ray of two patients submitted to internal fixation using a femoral intramedullary nail (Gamma 3 nail, Stryker ${ }^{\circledR}$ ). Both patients presented an imminent or pathological fracture due to bone metastases of different primary tumors [16].
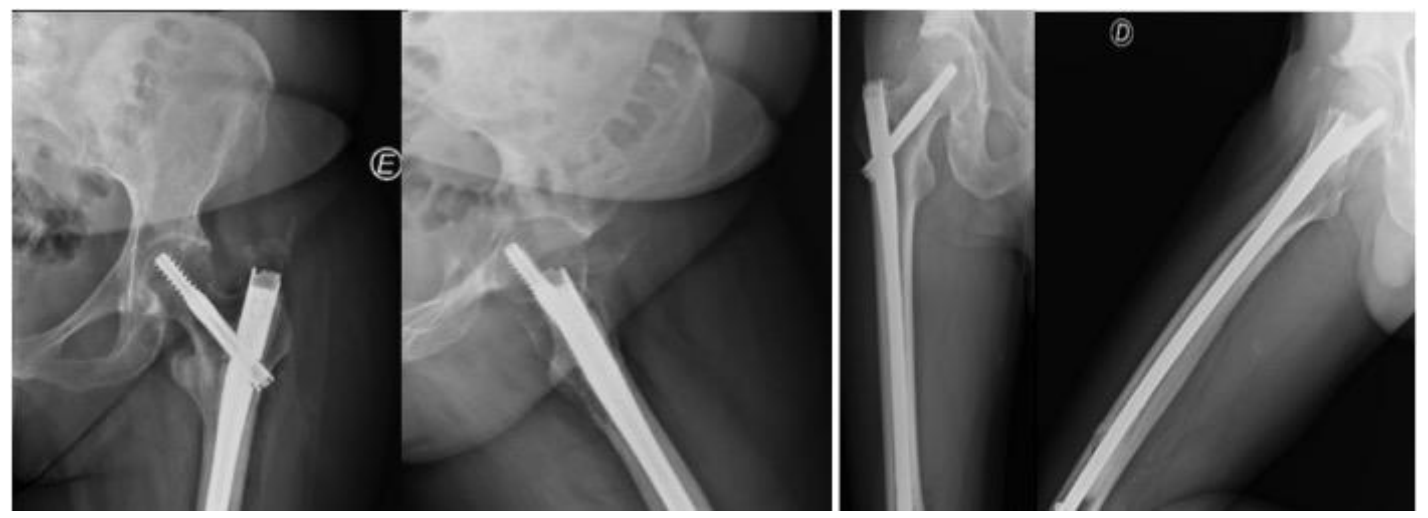

Fig. 1 Internal fixation with intramedullary nailing of femur.

Two computational models were reproduced without a femoral intramedullary system and other two extra models with an intramedullary nail with a diameter equal to $11 \mathrm{~mm}$. In the middle of the model, bone cement was introduced to fill in a metastatic lytic lesion area minimization with the dimensions equal to $20 \mathrm{~mm}$ in depth and variable width of 15 and $25 \mathrm{~mm}$. The bone lytic lesions were filled with bone cement. When the intramedullary systems are included, the cement material was spread in the same amount of affected area through the spongy bone. The numerical models were produced representing two-dimensional bone geometry with an external diameter equal to $31.2 \mathrm{~mm}$ and with cortical thickness of $7.35 \mathrm{~mm}$. In the middle length of the model a cement bone was introduced with the dimensions equal to $20 \mathrm{~mm}$ in depth and variable width 15 and $25 \mathrm{~mm}$. Numerical models were built accordingly to average dimensions obtained from digital medical images from patients in CHUP-ICBAS and agreed with a biomechanical data control group [17].

\begin{tabular}{|c|c|c|}
\hline Models: & Width cement $15 \times 20 \mathrm{~mm}$ (thin mantle) & Width cement $25 \times 20 \mathrm{~mm}$ (thick mantle) \\
\hline without intramedullary nail & & \\
\hline $\begin{array}{l}\text { with titanium intramedullary } \\
\text { nail }\end{array}$ & & \\
\hline
\end{tabular}

Fig. 3 Numerical models without intramedullary nail and with intramedullary titanium nail.

Figure 3 represents the geometries in the study without and with the internal stabilization, where blue zone represents the cortical tissue, violet is the spongy bone, the red colour is the used cement and the intramedullary nail is in dark blue colour. The numerical simulations were performed using the finite element method with ANSYS Multiphysics software. The geometrical model was meshed with a 2D thermal solid element (PLANE 77) with 8 nodes 
and a single degree of freedom, temperature, at each node. All material properties (cortical, spongy bone, cement A or B and intramedullary titanium nail) are in accordance with the literature [2], [16] and summarized in Table 1.

Table 1 Properties of materials [2], [16].

\begin{tabular}{lccc}
\hline Material & Density, $\mathrm{kg} / \mathrm{m}^{3}$ & Thermal Conductivity, W/K.m & Specific heat, J/kgK \\
\hline Cortical Bone & 2100 & 0.38 & 1260 \\
Spongy Bone & 620 & 0.39 & 4926 \\
Cement A or B & 1100 & 0.20 & 2000 \\
Titanium & 8900 & 29.00 & 377 \\
\hline
\end{tabular}

The time-temperature depend effect in cement was introduced in the numerical model according to the experimental results collected from literature [3], [6] represented in figure 4. According to the cement polymerization, a total simulation time equal to 1800 seconds was established for the numerical model: 384 seconds of heating with a peak of temperature equal to $83^{\circ} \mathrm{C}$ and 1416 seconds of cooling for cement $\mathrm{A}$ and 600 seconds of heating with a peak of temperature equal to $68^{\circ} \mathrm{C}$ and 1200 seconds of cooling for cement B. During the cement exothermic stage, there is no significant intramedullary blood flow increase. Thus, the ending time of the numerical analysis was 1800 seconds with an incremental time step equal to 5 seconds.

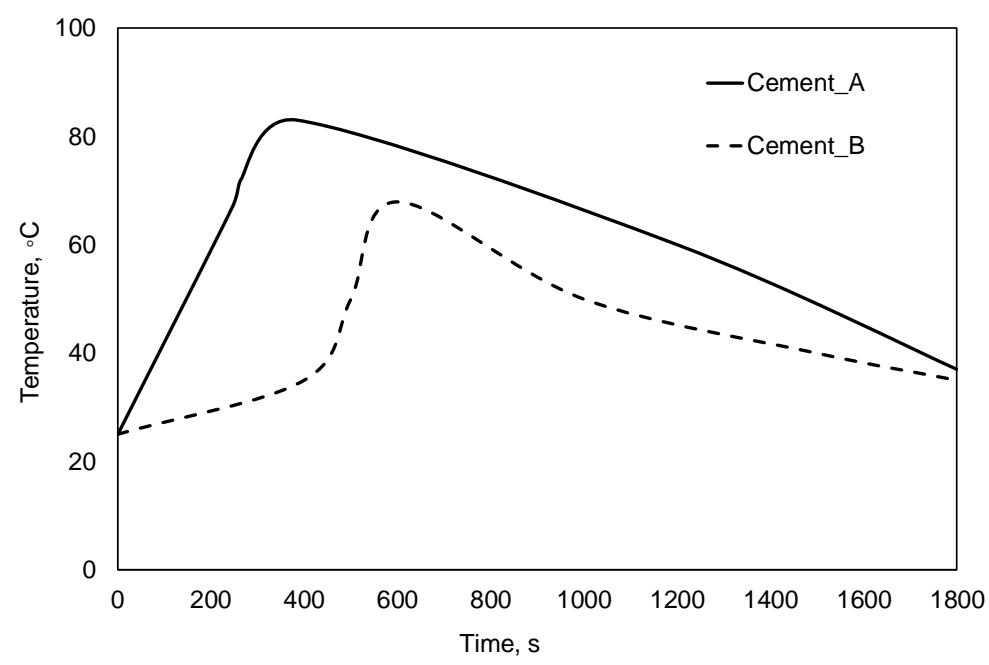

Fig. 4 Time-temperature curing effect of two bone cements

As seen in patients' imaging, the metastatic lytic lesion frequently affects spongy bone and one cortical bone (imminent or pathological fracture). For these models, a perfect contact was assumed between all interfaces. The cortical bone is a barrier for cement interdigitation independently of achieving cortical bone cells necrosis, due to its structural and physical properties. The bone cement fills in the lytic lesion area of the proximal femur minimization and easily interdigitate through the adjacent spongy bone with an expected higher porosity due to the non-blastic metastatic systemic disease. The pressure during the intramedullary titanium nail introduction, with an $11 \mathrm{~mm}$ diameter nail, compacts bone cement all around the nail through the spongy bone and until contact to both internal and external cortical bone, confirmed on patients imaging. We also assumed the heat transfer between different materials is performed exclusively by heat conduction. The boundary conditions considered in these simulations are the prescribed temperature in the bone cement region according the curing effect. The initial temperature in the model was assumed equal of $37^{\circ} \mathrm{C}$, mimicking the human body temperature.

\section{Results and Discussion}

The accuracy that can be obtained from finite element method is related to the mesh that is used. The presented results were obtained after a mesh convergence reducing the element size, that is an attractive approach due to its simplicity, validating and gaining confidence with the software and the obtained solution. The temperature field due to 
the cement type (A or B) curing effect is represented for the critical time equal to the maximum peak at $384 \mathrm{~s}$ and $600 \mathrm{~s}$, respectively, see figure 5 and 6 . Figure 5 represents the temperature field in the models without intramedullary nail system. The results show that the temperature in cement zone reaches the maximum value of $83^{\circ} \mathrm{C}$ when using cement type $\mathrm{A}$ and $68^{\circ} \mathrm{C}$ when using cement $\mathrm{B}$, and that the heat is spread through cortical and spongy bone. The thermal necrosis effect in bone tissue is represented in grey colour. Increasing the amount of the metastatic lytic lesion filled with cement type A (from 15 to $25 \mathrm{~mm}$ in width), the thermal necrosis increases the surrounding spongy region width by $10 \mathrm{~mm}$ equal in both models, increasing in lateral corners of the cement zone with more pronounced effect in depth. When using cement type B and with a mantle of 15 to $25 \mathrm{~mm}$ in width, the thermal necrosis region is reduced and the surrounding spongy region width is $5 \mathrm{~mm}$ equal in both models.

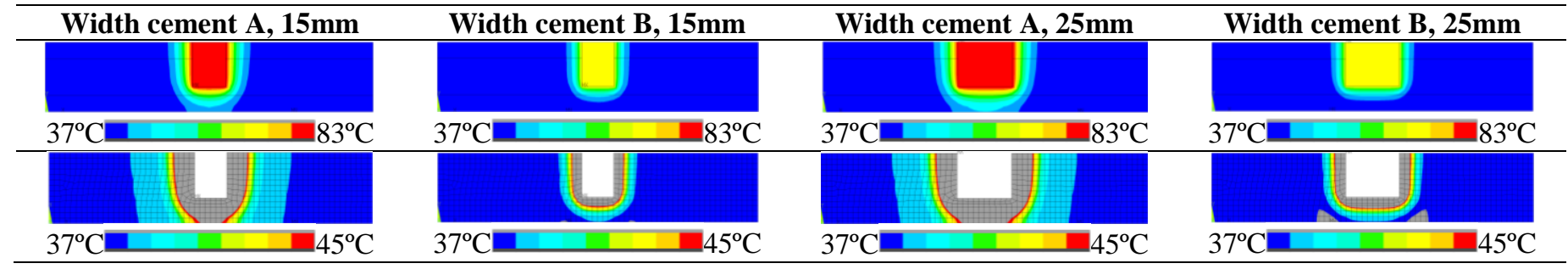

Fig. 5 Models without intramedullary nail, temperature at high peak cement polymerization.

Figure 6 represents the same computational models but including the intramedullary titanium nail, represented by the dark blue colour in figure 3 . The intramedullary fixation pressures the bone cement to fill from one cortical bone to another, around the nail. The temperature distribution is represented by the critical time (the peak temperature for each cement type polymerization). In the lytic lesion (and with the consequent increase of cement filling), the heat affects all bone tissue diameter due to the high thermal conductivity properties of the intramedullary titanium systems. All cement type A filling induced in the metastatic lytic lesion area affects the spongy bone and the cortical bone, and even small cement quantities produce high temperature in the surrounding region. When using cement type B the surrounding affected region is smaller, affecting only the adjacent area to the spongy bone. When comparing the temperature field between the two cement types used, cement A spread a higher necrosis effect and cement B has a pronounced effect only in the surrounding area to the cement or neighbour to the intramedullary nail. Bone necrosis was found in both models with either a thin or thick cement mantle.

\begin{tabular}{rllll}
\hline Width cement A, 15mm & Width cement B, 15mm & Width cement A, 25mm & Width cement B, 25mm \\
\hline $37^{\circ} \mathrm{C}$ & & & \\
\hline $37^{\circ} \mathrm{C}$ & & & \\
\hline
\end{tabular}

Fig. 6 Models with intramedullary titanium nail, temperature at high peak cement polymerization.

The results, obtained from the numerical analysis using the finite element method, allow to conclude about the high temperature spread in bone material. The presented results could play a key role in the multidisciplinary management of bone metastases. As refereed by [18] the cementoplasty can be used in difficult cases if there is good control of cement injection and real time quality imaging during the clinical procedure.

\section{Conclusions}

The temperature field located on the intramedullary system induces heat transfer in all the models due to higher heat conduction effect from the titanium material of the intramedullary nail, justified by the high material thermal conductivity. From the results, it can be concluded that with an increased lytic lesion and cement width, the thermal necrosis produces the same bone effect by adding the affected dimension by the amount of cement. There was a significant temperature difference between the different cement amounts used in this study due to the inclusion of the intramedullary nail, where the maximum temperatures at the bone increases significantly. The temperature at cementbone interface reaches the peak temperature values with both cement $\mathrm{A}$ and $\mathrm{B}$. When using cement type A, the 
surrounding necrotic cells above $45^{\circ} \mathrm{C}$ represent on average $10 \mathrm{~mm}$ of the bone layer. With cement type B only $5 \mathrm{~mm}$ on average of the bone layer is affected, representing $50 \%$ of the previous value. Nevertheless, the peak temperature between the cements used represents only a difference of $15^{\circ} \mathrm{C}$, corresponding to a reduction of $18 \%$ when using cement type $\mathrm{B}$, a high viscosity cement. As a general conclusion, bone cement filling and the structural stabilization with an intramedullary titanium nail have a synergic effect that can be applied to long bones metastatic lytic lesions treatment on a clinical practical application, either with low or high viscosity cement types.

\section{References}

[1] ESMO/European Sarcoma Network Working Group. Bone sarcomas: ESMO Clinical Practice Guidelines for diagnosis, treatment and follow-up. Annals of Oncology. 2014 Sep 1;25(suppl_3):iii113-23.

[2] Pérez MA, Nuño N, Madrala A, García-Aznar JM, Doblaré M. Computational modelling of bone cement polymerization: temperature and residual stresses. Computers in biology and medicine. 2009 Sep 1;39(9):751-9.

[3] Janssen D, Srinivasan P, Scheerlinck T, Verdonschot N. Effect of cementing technique and cement type on thermal necrosis in hip resurfacing arthroplasty - a numerical study. Journal of orthopaedic research. 2012 Mar;30(3):364-70.

[4] Huiskes R. Some fundamental aspects of human joint replacement: analyses of stresses and heat conduction in boneprosthesis structures. Acta Orthopaedica Scandinavica. 1980 Oct 1;51(sup185):3-208.

[5] Baliga BR, Rose PL, Ahmed AM. Thermal modeling of polymerizing polymethylmethacrylate, considering temperaturedependent heat generation.

[6] Santos Jr JG, Peixoto LS, Nele M, Melo PA, Pinto JC. Theoretical and Experimental Investigation of the Production of PMMA-Based Bone Cement. InMacromolecular Symposia 2006 Nov (Vol. 243, No. 1, pp. 1-12). Weinheim: WILEY-VCH Verlag.

[7] Fernandes MG, Fonseca EM, Teixeira TA, Jorge RN. Dynamic numerical simulation of different drill bit diameter on the polyurethane foams drilling. International Journal of Science and Technology. 2017;3(3):1-2.

[8] Fernandes MG, Fonseca EM, Natal R. Assessment of different drill diameter on bone drilling process. Journal of Mechanical Engineering and Biomechanics. 2017;1(6):135-41.

[9] Fernandes MG, Fonseca EM, Jorge RN, Vaz M, Dias MI. Thermal analysis in drilling of ex vivo bovine bones. Journal of Mechanics in Medicine and Biology. 2017 Aug 13;17(05):1750082.

[10] Tu YK, Chen LW, Ciou JS, Hsiao CK, Chen YC. Finite element simulations of bone temperature rise during bone drilling based on a bone analog. Journal of Medical and Biological Engineering. 2013 Jan 1;33(3):269-74.

[11] Eriksson AR, Albrektsson T. Temperature threshold levels for heat-induced bone tissue injury: a vital-microscopic study in the rabbit. Journal of prosthetic dentistry. 1983 Jul 1;50(1):101-7.

[12] Hillery MT, Shuaib I. Temperature effects in the drilling of human and bovine bone. Journal of Materials Processing Technology. 1999 Aug 30;92:302-8.

[13] Fernandes MG, Fonseca EM, Jorge RN, Manzanares C, Dias MI. Effect of drill speed on the strain distribution during drilling of bovine and human bones. Journal of Mechanical Engineering and Biomechanics. 2018;2(5):69-74.

[14] Eriksson RA, Albrektsson T. The effect of heat on bone regeneration: an experimental study in the rabbit using the bone growth chamber. Journal of Oral and Maxillofacial surgery. 1984 Nov 1;42(11):705-11.

[15] Gundapaneni D, Goswami T. Thermal isotherms in PMMA and cell necrosis during total hip arthroplasty. Journal of applied biomaterials \& functional materials. 2014 Sep;12(3):193-202.

[16] Oliveira VC, Fonseca EM. Computational model to predict the temperature distribution produced by bone cement. Journal of Mechanical Engineering and Biomechanics. 2018;3(2):8-13.

[17] Yang JH, Jung TG, Honnurappa AR, Cha JM, Ham CH, Kim TY, Suh SW. The analysis of biomechanical properties of proximal femur after implant removal. Applied bionics and biomechanics. 2016;2016.

[18] Deschamps F, De Baere T. Cementoplasty of bone metastases. Diagnostic and interventional imaging. 2012 Sep 1;93(9):685-9. 